\title{
The laboratory diagnosis of syphilis
}

\author{
Sam Ratnam PhD MPH FCCM
}

S Ratnam. The laboratory diagnosis of syphilis. Can J Infect Dis Med Microbiol 2005;16(1):45-51.

\begin{abstract}
Syphilis has several clinical manifestations, making laboratory testing a very important aspect of diagnosis. In North America, many unsuspected cases are discovered by laboratory testing. The etiological agent, Treponema pallidum, cannot be cultured, and there is no single optimal alternative test. Serological testing is the most frequently used approach in the laboratory diagnosis of syphilis. The present paper discusses the various serological and alternative tests currently available along with their limitations, and relates their results to the likely corresponding clinical stage of the disease. The need to use multiple tests is discussed, and the importance of quality control is noted. The complexity of syphilis serology means that the services of reference laboratories and clinical experts are often needed.
\end{abstract}

\section{Diagnostic de la syphilis en laboratoire}

La syphilis peut s'accompagner de manifestations cliniques variées, ce qui rend les analyses de laboratoire si importantes pour son diagnostic. En Amérique du Nord, beaucoup de cas insoupçonnés sont découverts lors d'analyses de laboratoire. L'agent causal, Treponema pallidum, ne peut pas être mis en culture et il n'existe aucun autre test idéal. Les épreuves sérologiques sont donc l'approche la plus souvent utilisée pour le diagnostic de la syphilis en laboratoire. Le présent article aborde les tests, sérologiques et autres, actuellement offerts et en décrit les limites; il établit en outre le lien entre les résultats des analyses et le stade probable de la maladie clinique. On mentionne également la nécessité d'utiliser plusieurs tests, de même que l'importance du contrôle de la qualité. Compte tenu de la complexité de la syphilis sur le plan de la sérologie, les services des laboratoires de référence et des experts cliniques sont souvent mis à contribution.

Key Words: Canada; Laboratory diagnosis; Nontreponemal tests; Serology; Syphilis; Treponema pallidum; Treponemal tests

Ts

he etiological agent of syphilis is Treponema pallidum.

Syphilis has diverse clinical manifestations and shares many clinical features with other treponemal and nontreponemal diseases. Therefore, it is mandatory that the clinical diagnosis is always supported by appropriate laboratory tests and that the test results are interpreted with reference to the patient's history and physical examination findings. Syphilis progresses through distinct primary, secondary, latent and tertiary stages. The ulcers that appear in primary and secondary syphilis are rich in treponemes; venereal transmission occurs through direct contact with these lesions. The stage of the disease at which the patient presents has implications for diagnosis and treatment. In some stages, the disease may be asymptomatic, and there are problems in diagnosing very early syphilis, neurosyphilis, asymptomatic congenital syphilis and syphilis in intravenous drug users and persons coinfected with serologically cross-reacting agents and HIV.

Although $T$ pallidum cannot be grown in culture, there are many tests for the direct and indirect diagnosis of syphilis. Still, there is no single optimal test. Direct diagnostic methods include the detection of $T$ pallidum by microscopic examination of fluid or smears from lesions, histological examination of tissues or nucleic acid amplification methods such as polymerase chain reaction (PCR). Indirect diagnosis is based on serological tests for the detection of antibodies. Serological tests fall into two categories: nontreponemal tests for screening, and treponemal tests for confirmation (Figure 1). All nontreponemal tests measure both immunoglobulin (Ig) G and IgM antiphospholipid antibodies formed by the host in response to lipoidal material released by damaged host cells early in infection and lipid from the cell surfaces of the treponeme itself. All treponemal tests use $T$ pallidum or its components as the antigen. If lesion exudate or tissue is available, direct examination is performed, followed by a nontreponemal serology test. A reactive nontreponemal test is then confirmed by a treponemal test. A confirmed serological test result is indicative of the presence of treponemal antibodies but does not indicate the stage of disease and, depending on the test, may not differentiate between past and current infection. Despite their shortcomings and the complexity of interpretation, serological tests are the mainstay in the diagnosis and follow-up of syphilis. Latent syphilis can only be diagnosed by serological tests. In fact, in North America, the majority of syphilis cases are identified at the latent stage by serological tests. The sensitivity and specificity of serological tests vary depending on the type of test and stage of the disease (Table 1). The application of various diagnostic tests in different stages of syphilis and their interpretation are summarized in Tables 2 and 3, respectively. There are no reliable routine laboratory methods to distinguish the other pathogenic treponemes from each other or from syphilis. Detailed descriptions of 


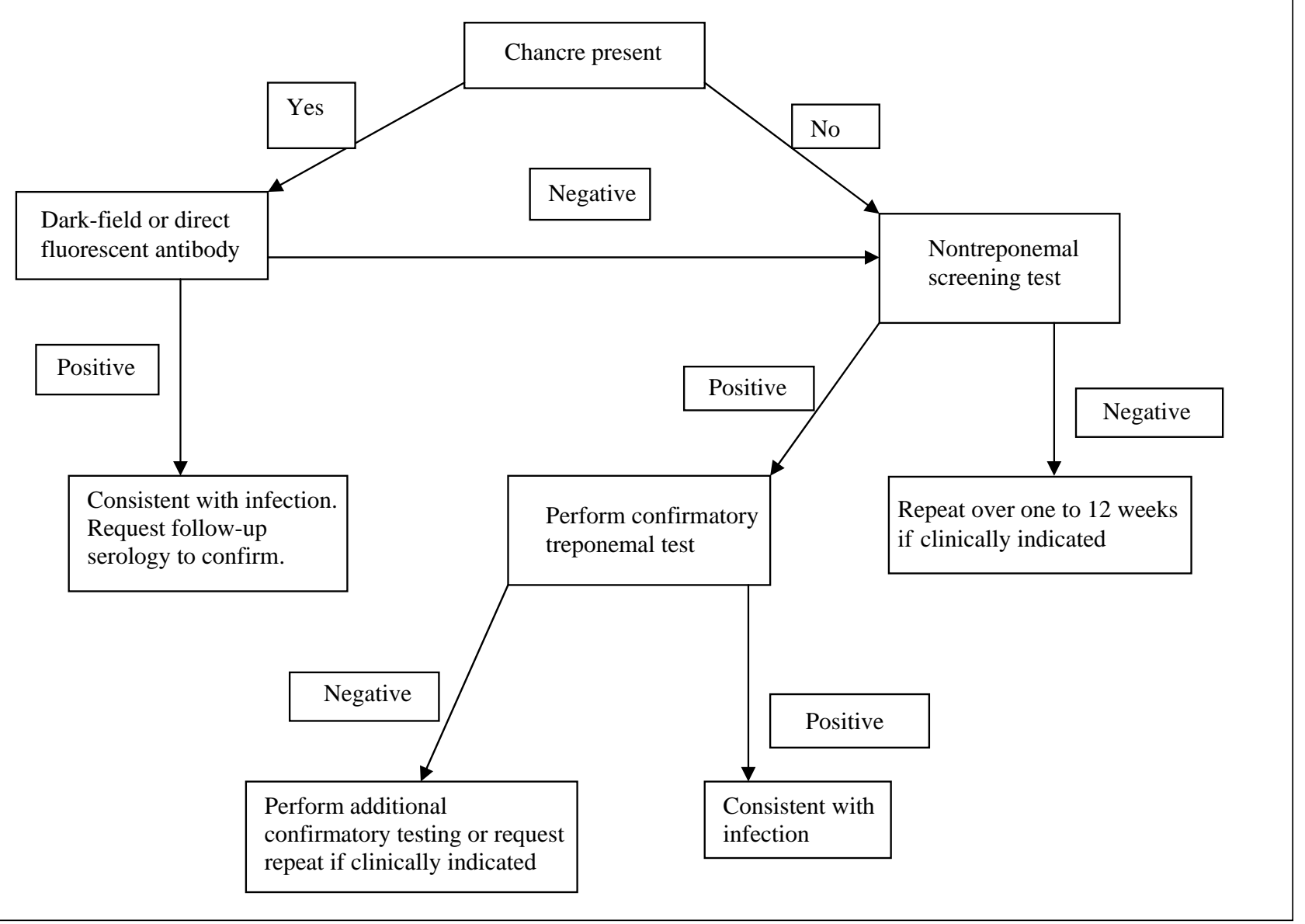

Figure 1) Testing algorithm for primary syphilis

TABLE 1

Performance of serological tests for syphilis

\begin{tabular}{lccccc}
\hline & \multicolumn{4}{c}{$\begin{array}{c}\text { Percentage of sensitivity by } \\
\text { stage of untreated syphilis }\end{array}$} \\
\cline { 2 - 5 } Test & Primary & Secondary & Latent & Late & Specificity \\
\hline VDRL & $78(74-87)^{*}$ & 100 & $96(88-100)$ & $71(34-94)$ & $98(96-99)$ \\
RPR card & $86(77-99)$ & 100 & $98(95-100)$ & 73 & $98(93-99)$ \\
USR & $80(72-88)$ & 100 & $95(88-100)$ & & 99 \\
TRUST & $85(77-86)$ & 100 & $98(95-100)$ & & $99(98-99)$ \\
FTA-ABS & $84(70-100)$ & 100 & 100 & 96 & $97(84-100)$ \\
FTA-ABS DS & $80(70-100)$ & 100 & 100 & & $98(97-100)$ \\
TP-PA ${ }^{\dagger}$ & $88(86-100)$ & 100 & 100 & & $96(95-100)$ \\
\hline
\end{tabular}

${ }^{*}$ Range in Centers for Disease Control and Prevention studies (1): †V Pope, Centers for Disease Control and Prevention, personal communication. DS Double staining; FTA-ABS Fluorescent treponemal antibody absorption; RPR Rapid plasma reagin; TP-PA Treponema pallidum particle agglutination; TRUST Toluidine red unheated serum test; USR Unheated serum reagin; VDRL Venereal Disease Research Laboratory

commonly used diagnostic procedures for syphilis can be found in current editions of laboratory manuals $(1,2)$.

\section{SPECIMEN OF CHOICE}

For direct examination, exudates from lesions of primary, secondary and early congenital syphilis are the most useful. It is important to collect clear, serous fluid free of erythrocytes, tissue debris and other organisms. Gentle abrasion of lesions may be necessary to express clear, serous fluid. Lesions should be cleansed with saline or water before collecting the specimen. This is especially important when collecting specimens from areas such as under the prepuce, where nonpathogenic treponemes may be present. For dark-field microscopy, the fluid should be collected on a slide, covered with a coverslip and examined within $20 \mathrm{~min}$. For direct fluorescent antibody testing, a smear should be made on a slide and then air dried.

While plasma can be used in some nontreponemal serological tests, serum is the specimen of choice for both nontreponemal and treponemal serological tests. Specimens that are hemolyzed, contaminated with bacteria, chylous, turbid or contain particulate matter are unsatisfactory. Care should be taken in shipping whole blood specimens to prevent hemolysis. Cerebrospinal fluid (CSF) testing is indicated in congenital and tertiary syphilis and when neurological symptoms are present. Blood contamination of CSF must be avoided because it may lead to false-positive CSF results. In congenital syphilis, venous samples from the mother and the child should be tested.

\section{TESTS FOR DIRECT DETECTION OF T PALLIDUM}

Dark-field microscopy

This method still remains one of the simplest and most reliable for the direct detection of $T$ pallidum. Exudates and fluids from lesions are examined as a wet mount using dark-field 
TABLE 2

The application and limitations of diagnostic tests in different stages of syphilis

\begin{tabular}{lll}
\hline Stage & Recommended tests & Comments \\
\hline Primary syphilis & Direct examination, & Detection of Treponema pallidum in lesions is definitive evidence of syphilis but a negative result does not rule \\
& $\begin{array}{l}\text { Nontreponemal tests, } \\
\text { out syphilis. PCR-based tests have a high reliability. In the first two to three weeks, serology may not be }\end{array}$ & positive in most cases, and in early primary syphilis, treponemal tests are recommended. The presence of \\
& a genital ulcer and a positive nontreponemal test may not indicate primary syphilis. Repeat serology over a \\
& two to 12 week period to rule out syphilis.
\end{tabular}

$\begin{array}{ll}\text { Secondary syphilis } & \begin{array}{l}\text { Direct examination, } \\ \text { Nontreponemal tests, }\end{array} \\ & \text { Trepollidum can be detected in skin and mucosal lesions, and PCR-based tests may be useful in atypical } \\ & \text { lesions. Serological tests have nearly } 100 \% \text { sensitivity. In persons with a history of syphilis, a fourfold increase }\end{array}$

Latent syphilis Nontreponemal tests, Nontreponemal tests are reactive in early latent syphilis but the sensitivity declines over time. In low Treponemal tests prevalence populations, false-positive results are common with both types of tests. Reactive treponemal tests in the absence of a reactive nontreponemal test require confirmation.

Tertiary syphilis Nontreponemal tests, Treponemal tests

Neurosyphilis

Congenital syphilis
Nontreponemal tests, Treponemal tests
Up to $30 \%$ may not be reactive in nontreponemal tests, whereas treponemal tests are almost always reactive. Therefore, treponemal tests should always be considered. Lesions are not suitable for direct microscopic examination.

Diagnosis requires a combination of tests. VDRL-CSF, the standard serological test for CSF, is highly specific but insensitive. Therefore, a negative VDRL-CSF result does not rule out neurosyphilis. In addition to a reactive VDRL-CSF, diagnosis depends on reactive serological tests and CSF abnormalities. FTA-ABS is more sensitive than VDRL-CSF but less specific. Therefore, the CSF FTA-ABS test may be useful to exclude neurosyphilis. PCR-based tests have a high reliability.

Diagnosis requires a combination of tests. Venous blood from both the mother and the child should be tested Serological tests on infant serum can be nonreactive if the mother has a low titre or was infected late in pregnancy. Immunoglobulin M-specific tests are useful for neonatal serum, but negative results may not rule out congenital syphilis. $T$ pallidum can be detected by direct examination of a variety of specimens from the neonate, and PCR-based tests have a high reliability. Asymptomatic congenital syphilis requires a comprehensive approach.

CSF Cerebrospinal fluid; FTA-ABS Fluorescent treponemal antibody absorption; PCR Polymerase chain reaction; VDRL Venereal Disease Research Laboratory

microscopy. The identification of $T$ pallidum is based on the characteristic morphology and motility of the spirochete. This method is suitable when the lesions are moist, and the examination can be done immediately after specimen collection. During the primary stage, serous fluid from the lesion contains numerous treponemes and, therefore, this approach is particularly useful in patients with immunodeficiency or in early syphilis when antibodies are not yet detectable. However, this technique requires a trained, experienced microscopist. Success is dependent on a number of factors, including too little or too much fluid on the slide, the presence of refractile elements in the specimen, improper thickness of the slide or cover slip, etc. Treatment with antibiotics may result in a false-negative finding. Therefore, although the demonstration of $T$ pallidum is the definitive method of diagnosis, dark-field microscopy has limited sensitivity, and failure to detect $T$ pallidum by this test does not rule out syphilis.

\section{Direct fluorescent antibody test for $\mathrm{T}$ pallidum}

The direct fluorescent antibody test for $T$ pallidum is easier to perform than dark-field microscopy. It detects antigen and, thus, does not require the presence of motile treponemes. It is the most specific test for the diagnosis of syphilis when lesions are present. This test uses fluorescein isothiocyanate-labelled antibody specific to pathogenic treponemes, and therefore is suitable for the examination of specimens from oral and rectal lesions. However, this test does not differentiate between $T$ pallidum and other pathogenic treponemes causing yaws, endemic syphilis and pinta.

Direct tests for $T$ pallidum in tissue sections

The direct fluorescent antibody tissue test detects $T$ pallidum in tissue sections and can be used in combination with a histological staining (1). T pallidum antibody-based nonfluorescent stain such as the IHC stain (Dako Corporation, California) can also be used (2). The latter offers the advantage of hematoxylin counterstain to simultaneously examine tissue structures. Silver staining, while useful, is nonspecific.

Nucleic acid amplification methods

A number of PCR-based methods have been developed for the detection of $T$ pallidum in clinical specimens (3-6). Although these methods are not standardized, they have been found to be highly sensitive, able to detect as low as one to 10 organisms per specimen with high specificity. These methods are also the most practical in certain settings (7). PCR undoubtedly holds promise as a test of choice for congenital syphilis, neurosyphilis and early primary syphilis when traditional tests have limited sensitivity. This method could be used to monitor treatment, and there is also potential to use it to differentiate new infections from old infections (1). While 
TABLE 3

Interpretation of serological tests for syphilis

\begin{tabular}{ccl}
\hline $\begin{array}{c}\text { Nontreponemal } \\
\text { tests }\end{array}$ & $\begin{array}{c}\text { Treponemal } \\
\text { tests }\end{array}$ & $\begin{array}{l}\text { Likely } \\
\text { interpretation }\end{array}$ \\
\hline+ & + & Syphilis, yaws or pinta \\
+ & - & False positive - No syphilis \\
- & + & Primary or latent syphilis; previously \\
treated or untreated syphilis; yaws or \\
& & pinta \\
- & - & No syphilis; incubating syphilis \\
\hline
\end{tabular}

\section{TABLE 4}

Causes of false-positive serological tests for syphilis

\begin{tabular}{ll}
\hline Nontreponemal tests & Treponemal tests \\
\hline Advancing age & Advancing age \\
Bacterial endocarditis & Brucellosis \\
Brucellosis & Cirrhosis \\
Chancroid & Drug addiction \\
Chickenpox & Genital herpes \\
Drug addiction & Hyperglobulinemia \\
Hepatitis & Immunizations \\
Idiopathic thrombocytopenic purpura & Infectious mononucleosis \\
Immunizations & Leptospirosis \\
Immunoglobulin abnormalities & Leprosy \\
Infectious mononucleosis & Lyme disease \\
Intravenous drug use & Malaria \\
Leprosy & Pinta \\
Lymphogranuloma venereum & Pregnancy \\
Malignancy & Relapsing fever \\
Measles & Scleroderma \\
Mumps & Systemic lupus erythematosus \\
Pinta & Thyroiditis \\
Pneumococcal pneumonia & Yaws \\
Polyarteritis nodosa & \\
Pregnancy & \\
Rheumatoid arthritis & \\
Rheumatic heart disease & \\
Rickettsial disease & \\
Systemic lupus erythematosus & \\
Thyroiditis & \\
Tuberculosis & \\
Ulcerative colitis & \\
Vasculitis & \\
Viral pneumonia & \\
Yaws & \\
\hline
\end{tabular}

there are no commercially available PCR-based test kits at the time of writing, this service may be available through select laboratories.

\section{NONTREPONEMAL SEROLOGICAL TESTS}

Nontreponemal tests become positive one to four weeks after the appearance of the primary chancre, and six weeks after exposure. These tests use the basic antigen formula of the Venereal Disease Research Laboratory (VDRL) test, which contains standardized amounts of cardiolipin, cholesterol and lecithin. The
Centers for Disease Control and Prevention (CDC)-approved standard tests include the VDRL slide test, the rapid plasma reagin (RPR) card test, the unheated serum reagin (USR) test and the toluidine red unheated serum test (TRUST) (1). Nontreponemal tests are rapid, simple and inexpensive. They are the only tests recommended to monitor the course of disease during and after treatment. Nontreponemal tests can also serve to detect reinfection. The main limitations of nontreponemal tests are their reduced sensitivity in primary syphilis and late latent syphilis (Table 1), false-positive results due to crossreactivity (Table 4), and the potential for false-negative results due to prozone reactions. Prozone reactions are falsenegative reactions that occur due to interference by high concentrations of target antibodies in a specimen. The disproportionate antibody-to-antigen ratio results in a 'rough' nonreactive or a very weakly reactive reaction. Such specimens will give a clearly positive reaction when diluted and retested, a process that brings the antibody-to-antigen ratio within the optimal range.

\section{VDRL, RPR, USR and TRUST tests}

The VDRL and USR tests are microflocculation tests and are read under a microscope. A disadvantage of the VDRL test is that the antigen suspension must be prepared fresh daily, whereas the USR test uses a stabilized antigen. However, the VDRL test is the only nontreponemal test that can be used to test CSF due to the limited sensitivity and specificity of the other nontreponemal tests. The RPR and TRUST tests are macroscopic flocculation tests and require no microscope. The RPR test uses a stabilized suspension of VDRL antigen to which charcoal particles are added to aid in the visualization of the test reaction. The RPR test is one of the most commonly used nontreponemal tests, and is a simplified version of the VDRL test. In the TRUST test, particles of toluidine red are used in place of the charcoal particles of the RPR test. Each of the above tests can be used as a quantitative test. Quantitative tests allow for the establishment of a baseline titre, which allows evaluation of recent infection and response to treatment. This also allows for the detection of reinfection or relapse in persons with a persistently reactive titre. However, the numerical values obtained may vary between tests; thus, when a patient is being followed with serial titres, the same test and preferably the same laboratory should be used.

\section{TREPONEMAL SEROLOGICAL TESTS}

Treponemal tests may remain reactive for years with or without treatment, and treponemal test antibody titres correlate poorly with disease activity. Therefore, treponemal tests should not be used to evaluate response to therapy, relapse or reinfection in previously treated patients. Also, treponemal tests do not differentiate venereal syphilis from endemic syphilis (yaws and pinta). Treponemal tests are used mainly as confirmatory tests to verify reactivity in nontreponemal tests. However, in populations of low disease prevalence, treponemal tests can be used for screening, utilizing a rapid test or enzyme immunoassay (EIA) format. Then, all positive patients would either be treated presumptively because the serious consequences of untreated infection far outweigh the effect of overtreatment, or have a follow-up RPR or VDRL to determine if they have active infection before treatment. Treponemal tests are also used as diagnostic tests in patients with nonreactive nontreponemal tests but with signs and symptoms of late syphilis. Treponemal tests are technically more difficult to perform and more expensive 
than nontreponemal tests. As with nontreponemal tests, falsepositive reactions can occur with treponemal tests (Table 4). Nevertheless, in the absence of immunosuppression, a nonreactive treponemal test is indicative of no past or present infection. There are many treponemal tests currently available, and some of the most commonly used treponemal tests are listed below.

\section{Fluorescent treponemal antibody absorption test and} fluorescent treponemal antibody absorption double staining tests The fluorescent treponemal antibody absorption (FTA-ABS) test is an indirect fluorescent antibody technique. In this procedure, serum samples are pretreated with an absorbent to remove nonspecific antibodies. The FTA-ABS double staining test is a modification of the FTA-ABS test using a double staining procedure with the addition of a contrasting counterstain. While these tests are highly sensitive and specific, they may produce variable results due to variation in equipment, reagents and interpretation.

\section{TP-PA test}

The Treponema pallidum particle agglutination (TP-PA) test (Fujirebio Inc, Japan) is a qualitative assay for the detection of antibodies to $T$ pallidum in serum or plasma. This test is based on the agglutination of coloured particle carriers sensitized with $T$ pallidum antigen and has replaced its predecessor, the microhemagglutination assay - Treponema pallidum (MHA-TP, Fujirebio Inc, Japan). The TP-PA test uses the same treponemal antigen as the MHA-TP test but offers the advantage of gelatin particles instead of erythrocytes, thus eliminating nonspecific reactions with plasma samples. The TP-PA test is less expensive and less complicated than the FTA-ABS tests, and the results are read with the unaided eye. It is one of the more commonly used treponemal tests. A positive TP-PA test in conjunction with a positive nontreponemal test is indicative of current or past infection with $T$ pallidum. The TP-PA test found to be an appropriate substitute for the MHA-TP test (8) is as sensitive as the FTA-ABS test in primary syphilis and as useful as the RPR test in monitoring therapy (9).

\section{EIA}

A number of treponemal EIA tests are commercially available (10), including Captia syphilis G, Captia Syphilis M and Captia select Syph-G (Trinity Biotech, Ireland), SpiroTek syphilis test (Organon Teknika, USA), Enzygnost Syphilis (Dade Behring, Germany), and Bio-Rad Syphilis G (Bio-Rad Laboratories, USA). The Captia Syphilis G test detects IgG antibodies, may be used both as a screening test and a confirmatory test, and is an appropriate substitute for the MHA-TP test (8). The Captia Syphilis M test is designed to detect IgM antibodies in congenital syphilis, and may be more sensitive than the FTA-ABS 19S IgM test (11); it is also considered to be suitable for the detection of early syphilis. The SpiroTek syphilis test has the highest sensitivity of all treponemal tests (especially in untreated primary syphilis) and is recommended as a confirmatory test by the CDC (1). EIA tests have been shown to be equal to or better than FTA-ABS and TP-PA tests in overall sensitivity and specificity and are more useful in HIV coinfected individuals (12-14). In addition, EIAs are amenable to automation, removing the variation and subjectivity of the human reader. While a number of laboratories have switched over to the treponemal EIAs for screening, the drawback is that treponemal tests generally remain reactive for life in most patients as discussed in the 'Treponemal serological tests' section of the current paper, and this could be misleading in individuals who no longer have active disease. There are also other emerging issues. The sensitivity of EIAs may not be any greater than MHA-TP in primary syphilis, and an increasing proportion may have only the EIA reactive result (V Pope, CDC, personal communication). For this reason, the CDC is currently recommending that, if EIA is used for screening, then an RPR test should be performed on all EIA reactives, and a second treponemal test such as TP-PA or FTA-ABS should be used for confirmation if the RPR test is reactive.

\section{Western blot}

This is similar to the Western blot test used for confirmation of HIV antibodies, and provides a molecular characterization of the antibody response through the visualization of characteristic banding patterns. Western blot methods using whole cell lysate and recombinant antigens have been developed $(15,16)$. Antibody reactivity to some of the treponemal antigenic bands is highly specific for syphilis. This test can detect either IgG or IgM antibodies and is considered a very useful adjunct confirmatory test $(6,17)$. This technique is used in some laboratories to resolve questionable results obtained with other treponemal tests; this service may be available through select laboratories. Details of this technique are described elsewhere (1).

\section{Rapid tests}

Treponemal tests are also commercially available in formats that can be performed at the point of care. They are available either as agglutination tests using latex particles coated with treponemal antigen or as immunochromatographic strips on which a positive reaction appears as a coloured line. Most of these tests can be used with whole blood, serum or plasma. They can be stored at room temperature, are simple to perform, require minimal training and no equipment, and the results can be read visually in less than 30 min. Over 20 such tests are commercially available but none are approved for use in North America. The World Health Organization has evaluated a number of rapid tests (18), the results of which are available on the World Health Organization's Web site (19). Because rapid tests do not contain internal quality control, periodic external quality control using laboratory-based tests is recommended.

\section{FALSE-POSITIVE AND FALSE-NEGATIVE SEROLOGICAL REACTIONS}

The phospholipid antibodies detected by nontreponemal tests are not only produced in syphilis and other treponemal disease but also in response to a variety of conditions unrelated to syphilis. Therefore, false-positive nontreponemal test reactions can have multiple causes (Table 4). Their incidence is generally $1 \%$ to $2 \%$. The rate of false-positives during pregnancy is no greater than that seen in the general population, but is higher among intravenous drug users. Generally, up to $90 \%$ of falsepositive reactions have a titre of less than $1: 8$, and reactive nontreponemal tests with titres less than 1:8 and subsequent nonreactive treponemal tests are considered to be biological false-positive reactions. Chronic false-positive reactions persist for more than six months and are often associated with autoimmune disorders and chronic inflammatory conditions. False-positive reactions can also occur with treponemal tests but this is less common than with nontreponemal tests (Table 4). 
Both types of tests can also yield false-negative results due to the prozone phenomenon. Such false-negatives occur in $1 \%$ to $2 \%$ of patients, especially in pregnant women and HIV patients. Serum from such patients should be tested at a 1:16 dilution. This, however, requires that the laboratory is given the relevant information from the patient's history and clinical diagnosis.

\section{EVALUATION OF TREATMENT EFFICACY}

Definite criteria for treatment success or failure have not been established, and assessing response to treatment is often difficult (20). Also, treatment failure cannot be reliably distinguished from reinfection. Generally, the efficacy of therapy is monitored by a remission of symptoms and a decline in antibody titre as measured by nontreponemal tests. Sequential antibody titres should be measured using the same test in the same laboratory. A post-treatment fall in titre confirms response to treatment, and a rise in titre indicates treatment failure or reinfection. Typically, the phospholipid antibody titre declines by fourfold after three months (eg, from 1:32 to 1:8) and by eightfold after six months (eg, from 1:32 to $1: 4$ ) with standard therapy (21). However, there is evidence that $15 \%$ of treated patients with early syphilis will not show a fourfold decline in titre at one year post-treatment (20). The time for decline in antibody titre may be longer with the RPR test than with the VDRL test (21). HIV coinfection may delay the decline in titre both in primary and secondary syphilis (22). CDC guidelines suggest that failure to achieve a fourfold decline in antibody titre by six months after therapy for primary and secondary syphilis and within 12 to 24 months in latent syphilis with initial high titres identifies patients at risk for treatment failure (1). Seroreversion is a slow process that depends on the stage of the disease and a number of other conditions $(7,23)$. Therefore, the rate of decline in titre is more useful than seroreversion. To follow seroreversion, serum must be examined every three to six months for at least two years. After successful treatment, the RPR and VDRL tests usually become nonreactive after one year in patients with primary syphilis, after two years in patients with secondary syphilis, and after five years in latent syphilis (7). Regardless, in some patients, particularly those treated in the latent or late stages, nontreponemal antibodies can persist at a low titre for a long period of time, sometimes for life, and this is referred to as the "serofast reaction" $(24,25)$. On the other hand, even without treatment, a patient may become serologically negative after several years. The VDRL test may be negative in up to one-fourth of patients with untreated late syphilis (24). Treponemal antibody titres correlate poorly with disease activity and should not be used to monitor treatment. PCR may be useful to rule out persistent infection. However, IgM-specific treponemal tests may be useful for monitoring treatment status and response to therapy. Nevertheless, untreated and inadequately treated infections beyond early stages, as well as reinfections, cannot be excluded based on a negative IgM test.

\section{ANTIMICROBIAL SUSCEPTIBILITY TESTING}

Antimicrobial susceptibility testing is technically difficult. $T$ pallidum strains are uniformly susceptible to penicillin and its derivatives, which are the preferred antimicrobial agents for the treatment of syphilis. Regardless, a number of methods have been used to determine the susceptibilities of representative strains (2); this service may be available through specialized laboratories and reference centres.

\section{QUALITY CONTROL}

It is critical to ensure the reliability and intra- and interlaboratory reproducibility of tests. This can be accomplished through a number of quality control measures that range from maintaining a good basic laboratory practice to the use of standardized reagents, controls and techniques, strict adherence to test procedures, and participation in recognized proficiency testing programs. The quality control programs should be under active surveillance to facilitate prompt recognition and correction of any problems. A detailed description of general and specific quality control measures pertaining to various tests is provided elsewhere (1).

\section{BEST PRACTICE CONSIDERATIONS}

Best practice considerations are summarized in Table 2; as stated, these considerations should be guided by the indications for, and limitations of, the various tests. The direct detection of $T$ pallidum by microscopy has diagnostic and practical limitations, and may be technically challenging. Due to a low prevalence of syphilis in North America, most diagnostic laboratories are unlikely to have the necessary technical expertise to perform this testing. Therefore, testing should be limited to those laboratories with expertise and, where possible, the direct detection of $T$ pallidum by microscopy and PCR-based tests for direct detection should be obtained from appropriate laboratories. The use of only one type of serological test is insufficient for diagnosis; both nontreponemal and treponemal serological tests should be carried out in all clinically suspected cases. Nontreponemal serological tests are amenable to being performed in the hospital laboratory setting, and if testing volume demands, syphilis screening using an approved test should be performed. However, a strict quality control program should be maintained to ensure reliability and reproducibility of the tests. If strict quality control cannot be met, it is prudent to obtain syphilis screening services from a reference laboratory. All reactive nontreponemal results must be confirmed by a treponemal test. In some instances, the patient's clinical history may require that treponemal tests are performed on samples with nonreactive nontreponemal results. In Europe, syphilis screening with both nontreponemal and treponemal tests has been widely used for many years. Although this combination provides an excellent screen for all stages of syphilis with the exception of very early primary infection when the treponemal test may not yet be positive, the cost-benefit of this approach should be considered. The other option might be to use one of the newer EIA tests, which can serve as both a screening and a confirmatory test. Rapid treponemal tests such as the Syphilis fast (Diesse, Italy) may be useful for point-of-care confirmation of screen results (26). Regardless, unless the testing volume demands an on-site treponemal testing service, such testing should be limited to reference laboratories.

\section{REFERENCES}

1. Larsen SA, Pope V, Johnson RE, Kennedy EJ Jr. A Manual of Tests for Syphilis. Washington DC: American Public Health Association, 1998.

2. Norris SJ, Pope V, Johnson RE, Larsen SA. Treponema and other human host-associated spirochetes. In: Murray PR, Baron EJ, Jorgensen JH, Pfaller MA, Yolken RH, eds. Manual of Clinical Microbiology. Washington DC: American Society for Microbiology, 2003:955-971.

3. Noordhoek GT, Wolters EC, de Jonge ME, van Embden JD. Detection of polymerase chain reaction of Treponema pallidum DNA in cerebrospinal fluid from neurosyphilis patients before and after antibiotic treatment. J Clin Microbiol 1991;29:1976-84. 
4. Burstain JM, Grimprel E, Lukehart SA, Norgard MV, Radolf JD. Sensitive detection of Treponema pallidum by using the polymerase chain reaction. J Clin Microbiol 1991;29:62-9.

5. Wicher K, Noordhoek GT, Abbruscato F, Wicher V. Detection of Treponema pallidum in early syphilis by DNA amplification. J Clin Microbiol 1992;30:497-500.

6. Sanchez PJ, Wendel GD, Grimprel E, et al. Evaluation of molecular methodologies and rabbit infectivity testing for the diagnosis of congenital syphilis and neonatal central nervous system invasion by Treponema pallidum. J Infect Dis 1993;167:148-57.

7. Wicher K, Horowitz HW, Wicher V. Laboratory methods of diagnosis of syphilis for the beginning of the third millennium. Microbes Infect 1999;1:1035-49.

8. Pope V, Fears MB, Morrill WE, Castro A, Kikkert SE. Comparison of the Serodia Treponema pallidum particle agglutination, Captia Syphilis-G, and SpiroTek reagin II tests with standard test techniques for diagnosis of syphilis. J Clin Microbiol 2001;38:2543-5.

9. Castro RR, Prieto ES, Santo I, Azevedo J, Exposto FL. Evaluation of the passive particle agglutination test in the serodiagnosis and follow-up of syphilis. Am J Clin Pathol 2001;116:581-5.

10. Schmidt BL, Edjlalipour M, Luger A. Comparative evaluation of nine different enzyme-linked immunosorbent assays for determination of antibodies against Treponema pallidum in patients with primary syphilis. J Clin Microbiol 2000;38:1279-82.

11. Stoll BJ, Lee FK, Larsen S, et al. Clinical and serologic evaluation of neonates for congenital syphilis: A continuing diagnostic dilemma. J Infect Dis 1993;167:1093-9.

12. Nayar R, Campos JM. Evaluation of the DCL Syphilis-G enzyme immunoassay test kit for the serologic diagnosis of syphilis. Am J Clin Pathol 1993;99:282-5.

13. Young H, Moyes A, McMillan A, Patterson J. Enzyme immunoassay for anti-treponemal IgG: Screening or confirmatory test? J Clin Pathol 1992;45:37-41.

14. Gutierrez J, Vergara MJ, Soto MJ, Piedrola G, Maroto M. Clinical utility of a competitive ELISA to detect antibodies against Treponema pallidum. J Clin Lab Anal 2000;14:83-6.
15. Sambri V, Marangoni A, Eyer C, et al. Western immunoblotting with five Treponema pallidum recombinant antigens for serologic diagnosis of syphilis. Clin Diagn Lab Immunol 2001;8:534-9.

16. Backhouse JL, Nesteroff SI. Treponema pallidum western blot: Comparison with the FTA-ABS test as a confirmatory test for syphilis. Diagn Microbiol Infect Dis 2001;39:9-14.

17. Byrne RE, Laska S, Bell M, Larson D, Phillips J, Todd J. Evaluation of Treponema pallidum western immunoblot assay as a confirmatory test for syphilis. J Clin Microbiol 1992;30:115-22.

18. Peeling, Rosanna W, and Ye Htun. Diagnostic tools for preventing and managing maternal and congenital syphilis: An overview. Bull World Health Organ 2004;82:439-46. Also available on the Internet $<$ http://www.scielosp.org/scielo.php?script= sci_arttext\&pid=S0042-96862004000600010\&lng=en\& $\&$ rm $=$ iso>. ISSN 0042-9686> (Version current at November 30, 2004).

19. World Health Organization. Sexually transmitted diseases: Diagnostics initiative. <www.who.int/std_diagnostics> (Version current at November 30, 2004).

20. Centers for Disease Control and Prevention. Sexually transmitted diseases treatment guidelines 2002. MMWR Recomm Rep 2002;51:1-78.

21. Brown ST, Zaidi A, Larsen SA, Reynolds GH. Serological response to syphilis treatment. A new analysis of old data. JAMA 1985;253:1296-9.

22. Rolfs RT, Joesoef MR, Hendershot EF, et al. A randomized trial of enhanced therapy for early syphilis in patients with and without human immunodeficiency virus infection. The Syphilis and HIV Study Group. N Engl J Med 1997;337:307-14.

23. Romanowski B, Sutherland R, Fick GH, Mooney D, Love EJ. Serologic response to treatment of infectious syphilis. Ann Intern Med 1991;114:1005-9.

24. Hook EW 3rd, Marra CM. Acquired syphilis in adults. N Engl J Med 1992;326:1060-9.

25. Fiumara NJ. Serologic responses to treatment of 128 patients with late latent syphilis. Sex Transm Dis 1979;6:243-6.

26. Fears MB, Pope V. Syphilis fast latex agglutination test, a rapid confirmatory test. Clin Diagn Lab Immunol 2001;8:841-2. 


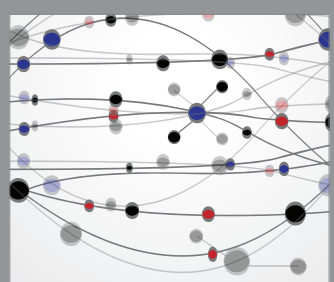

The Scientific World Journal
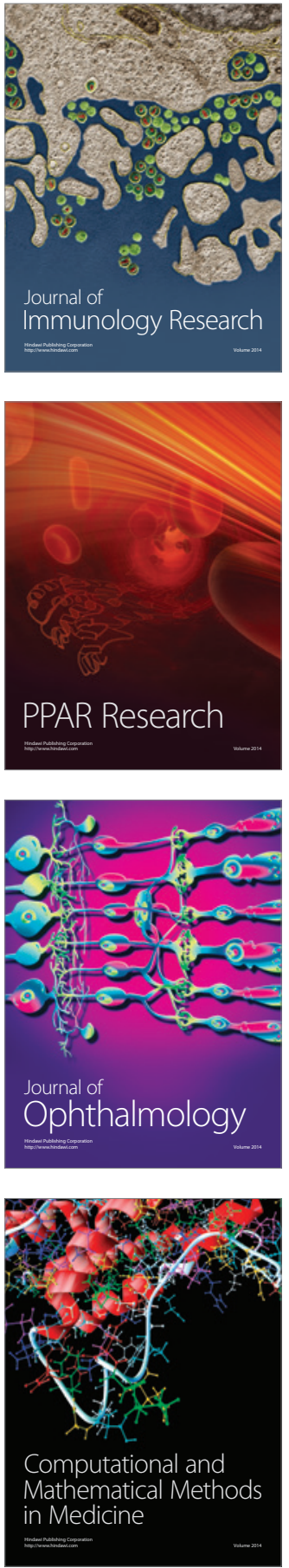

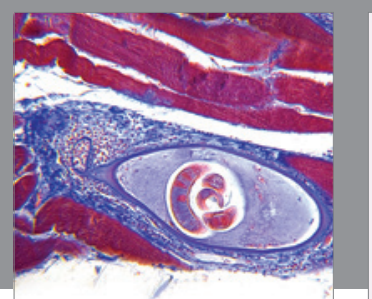

Gastroenterology Research and Practice

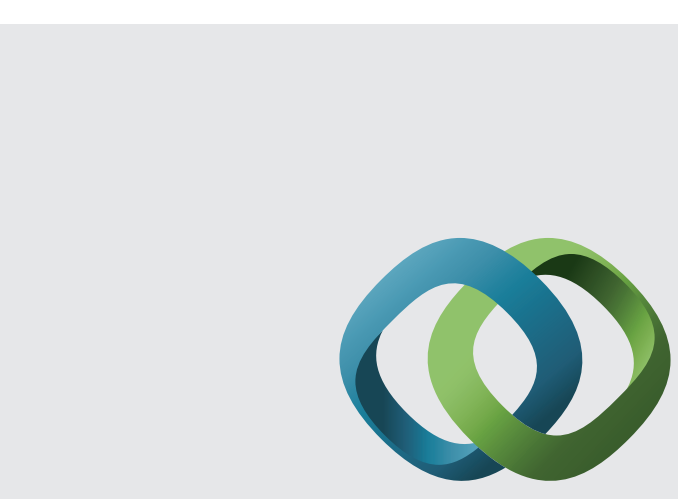

\section{Hindawi}

Submit your manuscripts at

http://www.hindawi.com
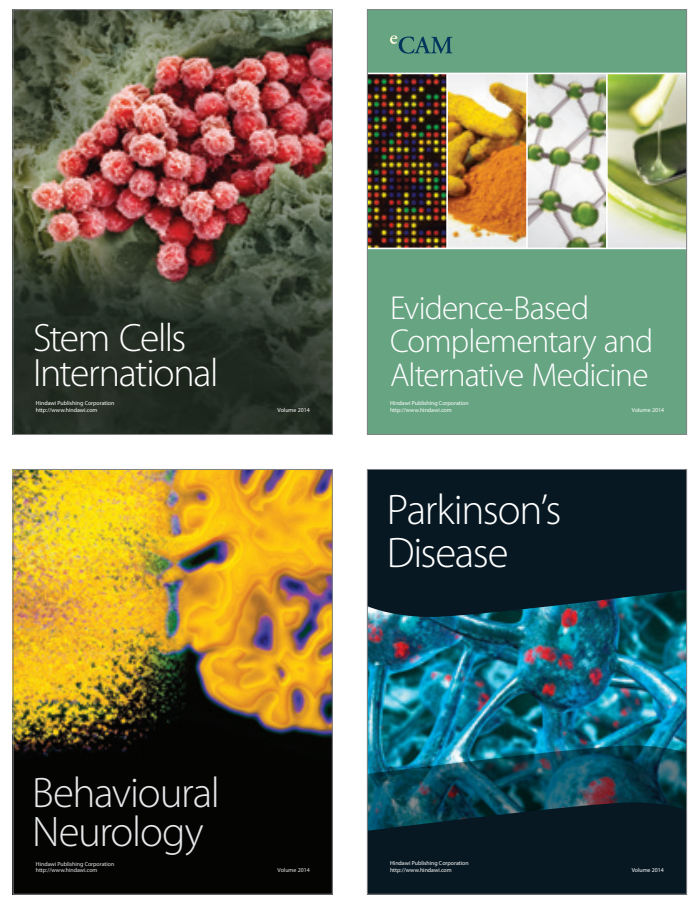
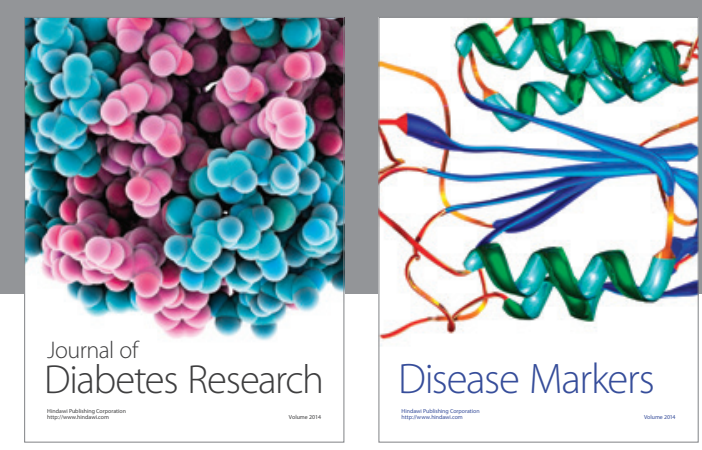

Disease Markers
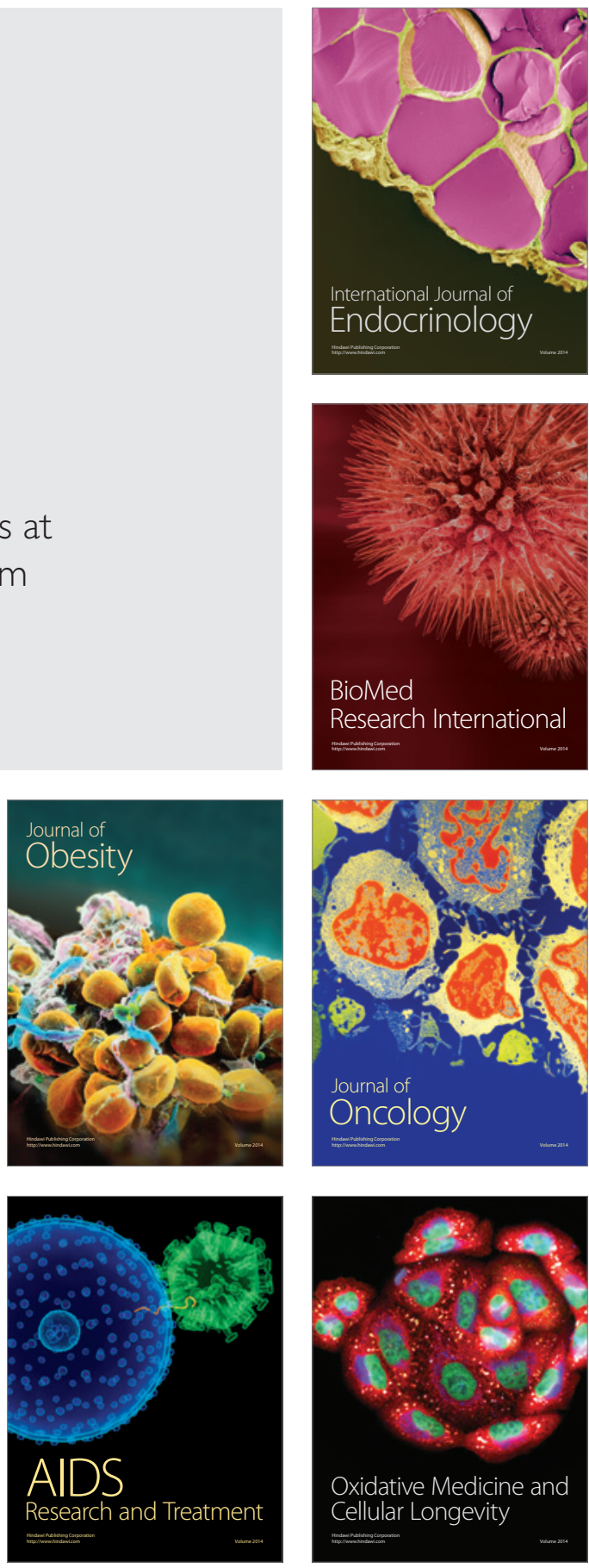